TRANSACTIONS OF THE

AMERICAN MATHEMATICAL SOCIETY

Volume 361, Number 4, April 2009, Pages 1749-1764

S 0002-9947(08)04781-8

Article electronically published on November 5, 2008

\title{
ON THE UNFOLDING OF SIMPLE CLOSED CURVES
}

\author{
JOHN PARDON
}

\begin{abstract}
We show that every rectifiable simple closed curve in the plane can be continuously deformed into a convex curve in a motion which preserves arc length and does not decrease the Euclidean distance between any pair of points on the curve. This result is obtained by approximating the curve with polygons and invoking the result of Connelly, Demaine, and Rote that such a motion exists for polygons. We also formulate a generalization of their program, thereby making steps toward a fully continuous proof of the result. To facilitate this, we generalize two of the primary tools used in their program: the Farkas Lemma of linear programming to Banach spaces and the Maxwell-Cremona Theorem of rigidity theory to apply to stresses represented by measures on the plane.
\end{abstract}

\section{INTRODUCTION}

Imagine a loop of string lying flat on a table without crossing itself. Now suppose the loop is slowly deformed until it becomes convex, without stretching or breaking it, in an expansive motion. By expansive, we mean that if you pick any pair of points on the string, then during the deformation, the distance between them will be nondecreasing. Then we can ask whether, given an initial loop, there always exists an expansive motion which deforms that loop until it becomes convex. If the loop is a polygon, then the answer is yes, as proved by Connelly, Demaine, and Rote 22. The first theorem of this paper (Theorem 2.2) is that the answer is yes for any rectifiable curve, no matter how complicated (in section 2.3, we give some examples of pathological curves to which the theorem applies). This solves Problem 4 listed by Ghomi [3, p. 1].

The proof of the main theorem uses a limiting process, relying on the result of 2]. We generalize the program used in [2], which relies on techniques of linear programming, specifically the Farkas Lemma. This approach naturally lends itself to computation; an example of research on the computation of nonexpansive unfoldings of polygons is given by [1. In a continuous analogue of the program, we develop a version of the Farkas Lemma for Banach spaces (Theorem 4.2) as well as a continuous version of the Maxwell-Cremona Theorem (Theorem 5.1), a combinatorial version of which was used in the program in [2]. A different version of the Farkas Lemma in Banach spaces and specifically in $L^{p}$ spaces has been studied in [4. We are not aware of any previous generalization of the Maxwell-Cremona Theorem to the case considered here. Finally, we use the continuous version of the program to give a different proof of the existence of infinitesimal expansions for

Received by the editors December 29, 2006.

2000 Mathematics Subject Classification. Primary 53C24; Secondary 53A04.

(C)2008 American Mathematical Society Reverts to public domain 28 years from publication 
polygons. The hope is that a continuous analogue of the discrete program could yield a direct proof (one which does not rely on approximation by polygons) of the main theorem for some class of curves more general than polygons.

The author would like to thank Robert Bryant for many useful conversations about the work in this paper, regarding both its content and presentation, and Robert Connelly for suggesting some reorganization to clarify the results. The author also thanks Andrew Ferrari for introducing him to many of the techniques used here.

1.1. Notation. We will use the following function spaces:

$C(X, Y)$ : the Banach space of continuous functions from $X$ to $Y$ given the supremum norm.

$C_{c}(X, Y)$ : the subspace of $C(X, Y)$ consisting of functions of compact support.

$C_{0}(X, Y)$ : the Banach space completion of $C_{c}(X, Y)$ with respect to the supremum norm. These are the functions that "vanish at infinity".

$C_{0}^{\infty}(X, Y)$ : the subspace of $C_{c}(X, Y)$ consisting of infinitely differentiable functions.

$L^{p}(X, Y)$ : the Banach space of $L^{p}$ functions from $X$ to $Y$.

If $Y$ is left out, it is assumed to be $\mathbb{R}$, except in section 2, where it is assumed to be $\mathbb{C}$. All Hilbert and Banach spaces are implicitly assumed to be over $\mathbb{R}$, except in section 2, where they will be over $\mathbb{C}$. If $E$ is a Banach space, $E^{*}$ is its dual. The duality bracket $\langle x, y\rangle$ will be used both in the case that $x \in E^{*}$ and $y \in E$, and in the case that $x, y \in H$, a Hilbert space. We will write $\mathcal{L}(X, Y)$ for the Banach space of bounded linear transformations from $X$ to $Y$ given the operator norm.

\section{Proof For General CURVES}

2.1. Preliminaries. Consider a simple closed curve in the plane. We wish to prove the existence of a continuous deformation of the curve into a convex curve, so that the intrinsic distance between every pair of points on the curve stays constant, and the extrinsic distance between every pair of points on the curve is nondecreasing. Here, by intrinsic distance we mean the distance along the curve, and by extrinsic distance, the Euclidean distance in $\mathbb{R}^{2}$.

A curve is called rectifiable if a finite intrinsic distance can be defined between every pair of points; that is, the supremum of the lengths of all inscribed polygons is finite:

$$
L_{x}^{y}(\mathbf{f}):=\sup _{x=a_{0}<a_{1}<\cdots<a_{k}=y} \sum_{j=1}^{k}\left|\mathbf{f}\left(a_{j}\right)-\mathbf{f}\left(a_{j-1}\right)\right|<\infty .
$$

We will only consider rectifiable curves in this paper. If a curve is rectifiable, then it has a unit speed parameterization; that is, $\mathbf{f}(s)=\int_{0}^{s} \mathbf{f}^{\prime}\left(s^{\prime}\right) d s^{\prime}$ and $\left|\mathbf{f}^{\prime}(s)\right|=1$ almost everywhere. Since a homothety will scale the arc length of a curve, it suffices to consider simple closed curves of length $2 \pi$. Thus, given $\mathbf{f}_{0}$, we seek a continuous family of simple closed curves $\mathbf{f}_{t}: \mathbb{R} / 2 \pi \longrightarrow \mathbb{R}^{2}$ parameterized by $t \in[0,1]$ such that each curve is of unit speed, $\left|\mathbf{f}_{t_{1}}(x)-\mathbf{f}_{t_{1}}(y)\right| \leq\left|\mathbf{f}_{t_{2}}(x)-\mathbf{f}_{t_{2}}(y)\right|$ whenever $t_{1} \leq t_{2}$, and $\mathbf{f}_{1}$ is convex. 
2.2. Main result. For this section, it will be natural to consider curves in $\mathbb{C}$ (rather than $\mathbb{R}^{2}$ ). Thus Banach spaces will be over $\mathbb{C}$. It will be convenient to have our curves reside in the following space:

$$
\mathcal{D}:=\left\{\mathbf{f}: \mathbb{R} / 2 \pi \longrightarrow \mathbb{C} \mid \mathbf{f}(0)=0, \mathbf{f} \text { absolutely continuous, } \mathbf{f}^{\prime} \in L^{\infty}(\mathbb{R} / 2 \pi)\right\} .
$$

There is, of course, the natural correspondence between $\mathbf{f} \in \mathcal{D}$ and $\mathbf{f}^{\prime} \in\{u \in$ $\left.L^{\infty}(\mathbb{R} / 2 \pi): \int u=0\right\}$. Thus $\mathcal{D}$ is a Banach space with norm $\left\|\mathbf{f}^{\prime}\right\|_{\infty}$. Now topologize $\mathcal{D}$ using the weak-* topology on $L^{\infty}(\mathbb{R} / 2 \pi)$. Since $L^{1}(\mathbb{R} / 2 \pi)$ is separable, the Banach-Alaoglu Theorem implies that any norm bounded sequence in $\mathcal{D}$ has a convergent subsequence. The choice of topology on $\mathcal{D}$ is justified by the following lemma.

Lemma 2.1. Suppose $\mathbf{f}_{n} \rightarrow \mathbf{f}$ in $\mathcal{D}$. Then $\mathbf{f}_{n} \rightarrow \mathbf{f}$ uniformly.

Proof. By the Uniform Boundedness Principle, we know that $\left\|\mathbf{f}_{n}\right\|$ is bounded. Thus there exists $M$ with $\left|\mathbf{f}_{n}^{\prime}\right| \leq M$; hence $\left\{\mathbf{f}_{n}\right\}$ is an equicontinuous family. It is clear that $\mathbf{f}_{n} \rightarrow \mathbf{f}$ pointwise since we have $\int \chi_{[0, x]} \mathbf{f}_{n}^{\prime} \rightarrow \int \chi_{[0, x]} \mathbf{f}^{\prime}$. Also, an equicontinuous sequence of functions converges pointwise if and only if it converges uniformly.

Define the continuous function $\mathcal{E}: \mathcal{D} \longrightarrow \mathbb{R}$ by $\mathcal{E}(\mathbf{f})=\iint_{(\mathbb{R} / 2 \pi)^{2}}|\mathbf{f}(x)-\mathbf{f}(y)|$. Also define the following order relation on $\mathcal{D}$ : we say that $\mathbf{f} \unlhd \mathbf{g}$ if and only if $|\mathbf{f}(x)-\mathbf{f}(y)| \leq|\mathbf{g}(x)-\mathbf{g}(y)|$ for all $x$ and $y$.

Theorem 2.2. Given a unit speed simple closed curve $\mathbf{f}: \mathbb{R} / 2 \pi \longrightarrow \mathbb{C}$, there exists a continuous function $\mathbf{h}:[0,1] \longrightarrow \mathcal{D}$ such that:

(1) $\mathbf{h}(0)=\mathbf{f}$.

(2) $\mathbf{h}(1)$ is convex.

(3) $\mathbf{h}(t)$ has unit speed for all $t$.

(4) If $t_{1} \leq t_{2}$, then $\mathbf{h}\left(t_{1}\right) \unlhd \mathbf{h}\left(t_{2}\right)$.

Proof. For $n \geq 3$, consider the polygon $\mathcal{P}_{n}$ inscribed in $\mathbf{f}$ which has $n$ vertices spaced out at multiples of $2 \pi / n$ starting at zero. Explicitly:

$$
\mathcal{P}_{n}(x):=\left(1-\left\{\frac{n x}{2 \pi}\right\}\right) \mathbf{f}\left(\frac{2 \pi}{n}\left\lfloor\frac{n x}{2 \pi}\right\rfloor\right)+\left\{\frac{n x}{2 \pi}\right\} \mathbf{f}\left(\frac{2 \pi}{n}\left(\left\lfloor\frac{n x}{2 \pi}\right\rfloor+1\right)\right) .
$$

This polygon may or may not be simple. It will, however, divide the plane into a finite number of simply connected regions. Let $\mathcal{P}_{n}^{\prime}$ be a constant speed $s_{n} \leq 1$ parameterization of the boundary of that region which has greatest area. Then let $\mathbf{h}_{n}:[0,1] \longrightarrow \mathcal{D}$ be continuous and satisfy:

$\left(1^{\prime}\right) \mathbf{h}_{n}(0)=\mathcal{P}_{n}^{\prime}$.

$\left(2^{\prime}\right) \mathbf{h}_{n}(1)$ is convex.

$\left(3^{\prime}\right) \mathbf{h}_{n}(t)$ has speed $s_{n} \leq 1$ for all $t$.

$\left(4^{\prime}\right)$ If $t_{1} \leq t_{2}$, then $\mathbf{h}_{n}\left(t_{1}\right) \unlhd \mathbf{h}_{n}\left(t_{2}\right)$.

$\left(5^{\prime}\right) \mathbf{h}_{n}(t)(\pi) \in \mathbb{R}_{>0}$ for all $t$.

$\left(6^{\prime}\right) \mathcal{E}\left(\mathbf{h}_{n}(t)\right)$ is a linear function of $t$.

The existence of an $\mathbf{h}_{n}$ satisfying $\left(1^{\prime}\right)-\left(4^{\prime}\right)$ is implied by Theorem 1 of [2, p. 207]. Condition $\left(5^{\prime}\right)$ can be achieved by properly rotating each curve. The motion of [2] is strictly expansive, so $\mathcal{E}\left(\mathbf{h}_{n}(t)\right)$ will be strictly increasing, so a simple reparameterization in $t$ suffices to make it linear and satisfy $\left(6^{\prime}\right)$. 
Let $\mathbb{Q}_{[0,1]}=\mathbb{Q} \cap[0,1]$. This set is countable; suppose $\left\{r_{i}\right\}_{i=1}^{\infty}$ is a counting of it. Let $\mathbf{h}_{n}^{(0)}=\mathbf{h}_{n}$. Inductively, let $\mathbf{h}_{n}^{(i)}$ be a subsequence of $\mathbf{h}_{n}^{(i-1)}$ such that $\mathbf{h}_{n}^{(i)}\left(r_{i}\right)$ converges. (Such a subsequence is guaranteed to exist since $\left\|\mathbf{h}_{n}^{(i)}\left(r_{i}\right)\right\|=s_{n}$ is bounded). Now $\mathbf{h}_{j}^{(j)}$ converges pointwise to a function $\tilde{\mathbf{h}}: \mathbb{Q}_{[0,1]} \longrightarrow \mathcal{D}$ which satisfies:

$\left(1^{\prime \prime}\right) \tilde{\mathbf{h}}(0)(\mathbb{R} / 2 \pi)=\mathbf{f}(\mathbb{R} / 2 \pi)$.

$\left(2^{\prime \prime}\right) \tilde{\mathbf{h}}(1)$ is convex.

$\left(3^{\prime \prime}\right) \tilde{\mathbf{h}}(t)$ has speed $\leq 1$.

$\left(4^{\prime \prime}\right)$ If $t_{1} \leq t_{2}$, then $\tilde{\mathbf{h}}\left(t_{1}\right) \unlhd \tilde{\mathbf{h}}\left(t_{2}\right)$.

$\left(5^{\prime \prime}\right) \tilde{\mathbf{h}}(t)(\pi) \in \mathbb{R}_{>0}$ for all $t$.

$\left(6^{\prime \prime}\right) \mathcal{E}(\tilde{\mathbf{h}}(t))$ is a linear function of $t$.

We will now construct $\mathbf{h}:[0,1] \longrightarrow \mathcal{D}$. For every $t \in[0,1]$, we set $\mathbf{h}(t)$ to be some arbitrary subsequential limit of $\tilde{\mathbf{h}}\left(q_{j}\right)$, where $q_{j}$ is some sequence of rationals converging to $t$. Clearly $\mathbf{h}$ satisfies $\left(1^{\prime \prime}\right)-\left(6^{\prime \prime}\right)$ as well. Now $\left(1^{\prime \prime}\right)$ and $\left(3^{\prime \prime}\right)$ together mean that $\mathbf{h}(0)(s)=\mathbf{f}(s+\Delta)$ for some $\Delta$. We can take $\Delta=0$. Hence we have (1), $(2)$, and (4). To prove (3), note that:

$$
\left|\frac{\mathbf{f}(x+h)-\mathbf{f}(x)}{h}\right| \leq\left|\frac{\mathbf{h}(t)(x+h)-\mathbf{h}(t)(x)}{h}\right| \leq 1 .
$$

As $h \rightarrow 0$, the left hand side approaches 1 for almost all $x$; hence $\left|\mathbf{h}(t)^{\prime}(x)\right|=1$ almost everywhere as desired.

Finally, we must show that $\mathbf{h}$ is in fact continuous. This follows from $\left(5^{\prime \prime}\right)$ and $\left(6^{\prime \prime}\right)$ in the following way. Suppose the contrary, that there is some $t$ where $\mathbf{h}$ is not continuous. Then there exists a sequence $q_{j} \rightarrow t$ with either $q_{j}<t$ for all $j$ or $q_{j}>t$ for all $j$, and a neighborhood $N$ of $\mathbf{h}(t)$ such that $\mathbf{h}\left(q_{j}\right) \notin N$ for all $j$. Now a subsequence of $\mathbf{h}\left(q_{j}\right)$ will converge in $\mathcal{D}$ to a limit $\mathbf{g}$. Now we have:

(i) $\mathcal{E}(\mathbf{g})=\mathcal{E}(\mathbf{h}(t))$.

(ii) $\mathbf{g} \unlhd \mathbf{h}(t)$ or $\mathbf{g} \unrhd \mathbf{h}(t)$ depending on whether $q_{j}<t$ or $q_{j}>t$.

(iii) $\mathbf{g}(0)=0=\mathbf{h}(t)(0)$.

(iv) $\mathbf{g}(\pi), \mathbf{h}(t)(\pi) \in \mathbb{R}_{>0}$.

The conditions (i) and (ii) imply that $|\mathbf{g}(x)-\mathbf{g}(y)|=|\mathbf{h}(t)(x)-\mathbf{h}(t)(y)|$ for all $x$ and $y$. This means that the curves are rigid motions of each other. Then (iii) and (iv) imply that they are actually the same curve since they have the same orientation. Thus a subsequence of $\mathbf{h}\left(q_{j}\right)$ converges to $\mathbf{h}(t)$. This is of course a contradiction since each $\mathbf{h}\left(q_{j}\right)$ is outside the neighborhood $N$ of $\mathbf{h}(t)$. This contradiction proves that $\mathbf{h}$ is continuous.

2.3. Pathological rectifiable curves. Define $f_{-}$and $f_{+}$:

$$
f_{ \pm}(x)= \begin{cases}x^{2} \sin x^{-1} \pm e^{-1 / x} & x>0 \\ 0 & x=0 .\end{cases}
$$

If we plot $f_{-}$and $f_{+}$on $\left[0, \pi^{-1}\right]$ and add line segments around the left side of the curve to close it, we get an infinite number of interlocking "teeth". This example is based on a polygon with a finite number of such teeth unfolded by Erik Demaine. 
We also have:

$$
g(t)= \begin{cases}t^{2} e^{i / t} & t>0, \\ 0 & t=0, \\ -t^{2} e^{-i / t} & t<0 .\end{cases}
$$

Plotting $g$ on $\left[-\pi^{-1}, \pi^{-1}\right]$ and adding line segments to close the curve gives a simple closed curve with an infinite spiral. By Theorem 2.2, both of these curves can be unfolded in an expansive motion, something which is not at all intuitive considering their geometry.

\section{A generalization of the CDR PROGRAm}

The program in 22 proves the existence of an infinitesimal expansion for any polygon. That is, if a nonconvex polygon has vertices $\mathbf{p}_{i}$, it shows the existence of velocities $\mathbf{v}_{i}$ satisfying:

$$
\begin{aligned}
\left(\mathbf{p}_{i}-\mathbf{p}_{i+1}\right) \cdot\left(\mathbf{v}_{i}-\mathbf{v}_{i+1}\right) & =0 \\
\left(\mathbf{p}_{i}-\mathbf{p}_{j}\right) \cdot\left(\mathbf{v}_{i}-\mathbf{v}_{j}\right) & >0 \text { for } i \text { and } j \text { not adjacent. }
\end{aligned}
$$

From this, it is relatively straightforward to solve a differential equation of the form $\frac{d}{d t}\left\{\mathbf{p}_{i}\right\}=\left\{\tilde{\mathbf{v}}_{i}\right\}$ (where the $\left\{\tilde{\mathbf{v}}_{i}\right\}$ depend continuously on the $\left\{\mathbf{p}_{i}\right\}$ ), thus constructing an expansive motion of the polygon. Clearly, if we have a curve $\mathbf{f}$, then the analogue is to find a variation $\varphi$ satisfying:

$$
\begin{aligned}
\mathbf{f}^{\prime}(x) \cdot \varphi^{\prime}(x) & =0 \text { for all } x, \\
(\mathbf{f}(x)-\mathbf{f}(y)) \cdot(\varphi(x)-\varphi(y)) & \geq 0 \text { for all } x \text { and } y .
\end{aligned}
$$

The generalized program developed here will be able to prove the existence of infinitesimal expansions for polygons, a hard theoretical result of [2]. It also proves the existence of "almost" expansive variations for all rectifiable curves which in a neighborhood of any point look like the rotated graph of a function from $\mathbb{R}$ to $\mathbb{R}$. By this we mean that for every $x \in \mathbb{R} / 2 \pi$, there exists $\mathbf{v} \in \mathbb{R}^{2}$ such that $\mathbf{f}(y) \cdot \mathbf{v}$ is one to one in a neighborhood of $x$. The final result of this generalized program is Theorem 3.4.

The generalizations of the Farkas Lemma and the Maxwell-Cremona Theorem, the tools used in the program, are stated and proved in sections 4 and 5 respectively.

3.1. Notation. Let $H:=\left\{u \in C\left(\mathbb{R} / 2 \pi, \mathbb{R}^{2}\right): u(0)=0, u\right.$ absolutely continuous, and $\left.\int\left|u^{\prime}\right|^{2}<\infty\right\}$. So that $H$ is a Hilbert space, equip it with the norm $\sqrt{\int\left|u^{\prime}\right|^{2}}$ and inner product $\int u^{\prime} \cdot v^{\prime}$. Topologize $H$ with the weak topology. We will need the sets:

$$
\begin{aligned}
Q_{\mathbf{f}} & :=\left\{u \in H: u^{\prime} \cdot \mathbf{f}^{\prime} \equiv 0\right\} \quad \text { (a closed subspace), } \\
T & :=\left\{t \in C\left((\mathbb{R} / 2 \pi)^{2}\right)^{*}: t \geq 0\right\} .
\end{aligned}
$$

Note that we will be looking for $\varphi \in Q_{\mathbf{f}}$, since it is these variations which preserve arc length. Also note that in this section, we do not assume that $\mathbf{f}$ is parameterized by arc length.

Lemma 3.1. If $\mathbf{g}_{n} \rightarrow \mathbf{g}$ in the weak topology on $H$, then $\mathbf{g}_{n} \rightarrow \mathbf{g}$ uniformly. 
Proof. This is completely analogous to Lemma 2.1. We know that $\mathbf{g}_{n} \rightarrow \mathbf{g}$ pointwise. Observing that $\left\|\mathbf{g}_{n}\right\|$ is bounded, we have the inequality:

$$
\int_{a}^{b}\left|\mathbf{g}_{n}^{\prime}\right|=\int_{\mathbb{R} / 2 \pi} \mathbf{g}_{n}^{\prime} \frac{\mathbf{g}_{n}^{\prime}}{\left|\mathbf{g}_{n}^{\prime}\right|} \chi_{[a, b]} \leq \sqrt{\int_{\mathbb{R} / 2 \pi}\left|\mathbf{g}_{n}^{\prime}\right|^{2}} \sqrt{\int_{a}^{b}\left|\frac{\mathbf{g}_{n}^{\prime}}{\left|\mathbf{g}_{n}^{\prime}\right|}\right|^{2}} \leq M \sqrt{b-a} .
$$

This shows that the $\mathbf{g}_{n}$ are uniformly continuous, and hence converge uniformly.

Define $D \subset H$, the set of curves we will consider, to be the set of $\mathbf{f} \in H$ satisfying:

(1) $\mathbf{f}$ is a simple closed curve, that is, $\mathbf{f}$ is injective.

(2) $\mathbf{f}^{\prime} \neq 0$ almost everywhere (this in fact is not implied by (1)).

(3) For every $x$, there exists $\delta>0$ and $\mathbf{v}$ such that $\mathbf{f}(y) \cdot \mathbf{v}$ is one to one for $|y-x|<\delta$ (locally graph-like).

The symbol $\mathbf{f}$ will always denote a member of $D$.

The following bounded operator will be essential to the program; it is called the Rigidity Operator:

$$
\begin{gathered}
R_{\mathbf{f}}: H \longrightarrow C\left((\mathbb{R} / 2 \pi)^{2}\right), \\
\left(R_{\mathbf{f}} \varphi\right)(x, y)=(\mathbf{f}(x)-\mathbf{f}(y)) \cdot(\varphi(x)-\varphi(y)) .
\end{gathered}
$$

3.2. Outline of the program. Before we state the final result of the program in its full generality (Theorem 3.4), it is useful to state the following corollary, which gives the general idea of the result.

Corollary 3.2. Let $\mathbf{f} \in D$ not be convex. Let $V \subset(\mathbb{R} / 2 \pi)^{2}$ be closed and have the property that for all $(x, y) \in V$, the line segment between $\mathbf{f}(x)$ and $\mathbf{f}(y)$ is not competely contained in $\mathbf{f}(\mathbb{R} / 2 \pi)$ (for example, if $\mathbf{f}$ has no straight sections, we can take $\left.V=\left\{(x, y) \in(\mathbb{R} / 2 \pi)^{2}:|x-y|>\epsilon\right\}\right)$. Then there exists a $\varphi \in Q_{\mathbf{f}}$ such that:

$$
(\mathbf{f}(x)-\mathbf{f}(y)) \cdot(\varphi(x)-\varphi(y))>0 \text { for all }(x, y) \in V .
$$

This result includes the result [2] of the existence of infinitesimal expansions for nonconvex polygons.

Corollary 3.3 (Theorem 3 of [2, p. 215]). If $\left\{\mathbf{p}_{i}\right\}$ is a nonconvex simple polygon with no straight vertices, then there exist $\left\{\mathbf{v}_{i}\right\}$ satisfying:

$$
\begin{aligned}
\left(\mathbf{p}_{i}-\mathbf{p}_{i+1}\right) \cdot\left(\mathbf{v}_{i}-\mathbf{v}_{i+1}\right) & =0, \\
\left(\mathbf{p}_{i}-\mathbf{p}_{j}\right) \cdot\left(\mathbf{v}_{i}-\mathbf{v}_{j}\right) & >0 \text { for } i \text { and } j \text { not adjacent. }
\end{aligned}
$$

Proof. Apply Corollary 3.2 to $\mathbf{f}=$ the polygon and:

$$
\begin{aligned}
V=\left\{(x, y) \in(\mathbb{R} / 2 \pi)^{2}:\right. & \text { there are two full edges separating } x \text { and } y \\
& \text { in both directions }\} .
\end{aligned}
$$

Then we have a $\varphi$. Set $\mathbf{v}_{i}=\varphi\left(\mathbf{f}^{-1}\left(\mathbf{p}_{i}\right)\right)$. Then $\left(\mathbf{p}_{i}-\mathbf{p}_{j}\right) \cdot\left(\mathbf{v}_{i}-\mathbf{v}_{j}\right)>0$ for $i$ and $j$ not adjacent is clear from (3.9). Now:

$$
\left(\mathbf{v}_{i+1}-\mathbf{v}_{i}\right) \cdot\left(\mathbf{p}_{i+1}-\mathbf{p}_{i}\right)=\int_{\mathbf{f}^{-1}\left(\mathbf{p}_{i}\right)}^{\mathbf{f}^{-1}\left(\mathbf{p}_{i+1}\right)} \varphi^{\prime}(x) \cdot\left(\mathbf{p}_{i+1}-\mathbf{p}_{i}\right) d x=0
$$

since $\varphi \in Q_{\mathbf{f}}$.

Theorem 3.4. the main result of the generalization of the program of [2], is essentially Corollary 3.2 made uniform over some suitable set of curves. 
Theorem 3.4 (Analogue of Theorem 3 of [2, p. 215]). Suppose $D_{1} \subset D$ is (weakly) closed and contains no convex curves, and that $V \subset D_{1} \times(\mathbb{R} / 2 \pi)^{2}$ is closed. Additionally, suppose that for every $(\mathbf{f}, x, y) \in V$, the line segment joining $\mathbf{f}(x)$ and $\mathbf{f}(y)$ is not completely contained in $\mathbf{f}(\mathbb{R} / 2 \pi)$. Then there exists $\epsilon>0$ such that for each $\mathbf{f} \in D_{1}$, there exists $\varphi \in Q_{\mathbf{f}}$ with:

(1) $\|\varphi\|=1$,

(2) $R_{\mathbf{f}} \varphi(x, y) \geq \epsilon$ whenever $(\mathbf{f}, x, y) \in V$.

We can see that Corollary 3.2 is obtained by taking $D_{1}$ to consist of a single curve. A corollary which does not lose the uniformity is the following:

Corollary 3.5. Suppose $D_{1} \subset D$ is weakly closed, contains no convex curves, and contains no curves with straight sections. Then for every $\delta>0$, there exists $\epsilon>0$ such that for every $\mathbf{f} \in D_{1}$, there exists $\varphi \in Q_{\mathbf{f}}$ satisfying:

(1) $\|\varphi\|=1$,

(2) $(\varphi(x)-\varphi(y)) \cdot(\mathbf{f}(x)-\mathbf{f}(y)) \geq \epsilon$ if $|x-y| \geq \delta$.

Proof. Choose $V=D_{1} \times\left\{(x, y) \in(\mathbb{R} / 2 \pi)^{2}:|x-y| \geq \delta\right\}$ and apply Theorem 3.4 .

The main difficulty in showing the existence of a $\varphi$ which is expansive for all pairs $x$ and $y$ is the fact that $V$ being closed is critical to the proof. Clearly $(\mathbf{f}, x, x)$ can never be in $V$ since then we would conclude that $(\varphi(x)-\varphi(x)) \cdot(\mathbf{f}(x)-\mathbf{f}(x))>0$. Hence, we must always exclude a neighborhood of the "diagonal" of $(\mathbb{R} / 2 \pi)^{2}$. This means that we will not have shown that $(\varphi(x)-\varphi(y)) \cdot(\mathbf{f}(x)-\mathbf{f}(y))>0$ for all pairs $x$ and $y$.

The following theorem is the essence of why expansive variations exist. It relies on the generalization of the Maxwell-Cremona Theorem (Theorem 5.1).

Theorem 3.6 (Analogue of Theorem 4 of [2, p. 216]). If $\mathbf{f} \in D$ and $t \in T$ such that $\left\langle t, R_{\mathbf{f}} \alpha\right\rangle=0$ for all $\alpha \in Q_{\mathbf{f}}$, then either:

(1) The curve $\mathbf{f}$ is convex.

OR

(2) For all $(x, y) \in \operatorname{supp} t$, the line segment connecting $\mathbf{f}(x)$ and $\mathbf{f}(y)$ is completely contained in $\mathbf{f}(\mathbb{R} / 2 \pi)$.

In the spirit of the generalization of the Farkas Lemma (Theorem 4.2), it is possible to prove that Theorem 3.6 implies Theorem 3.4.

Proposition 3.7 (Analogue of Lemma 3 of [2, p. 216]). Theorem [3.6 implies Theorem 3.4 .

\subsection{Proof of Theorem 3.6 .}

Proof. Suppose that we have some $\mathbf{f} \in D$ and $t \in T$ with $\left\langle t, R_{\mathbf{f}} \alpha\right\rangle=0$ for all $\alpha \in Q_{\mathbf{f}}$. Let $\hat{\mathbf{f}}^{\prime}$ denote $\mathbf{f}^{\prime} /\left|\mathbf{f}^{\prime}\right|$.

First, let us show that there exists $\beta \in L^{2}(\mathbb{R} / 2 \pi)$ such that:

$$
\langle t, R \alpha\rangle=\int_{\mathbb{R} / 2 \pi} \beta(x) \hat{\mathbf{f}}^{\prime}(x) \cdot \alpha^{\prime}(x) d x .
$$

Clearly there exists $\mu \in L^{2}\left(\mathbb{R} / 2 \pi, \mathbb{R}^{2}\right)$ such that $\langle t, R \alpha\rangle=\int_{\mathbb{R} / 2 \pi} \mu(x) \cdot \alpha^{\prime}(x) d x$. Now we can constrain $\mu$ as follows. For any $\lambda \in L^{2}(\mathbb{R} / 2 \pi)$ satisfying $\int \lambda \hat{\mathbf{f}}^{\prime}=0$, we 
know that:

$$
\int_{\mathbb{R} / 2 \pi}\left(\mu(x) \cdot i \hat{\mathbf{f}}^{\prime}(x)\right) \lambda(x) d x=0 .
$$

The set $H$ of such a $\lambda$ is of codimension 2 in $L^{2}(\mathbb{R} / 2 \pi)$. Now $\mu(x) \cdot i \hat{\mathbf{f}}^{\prime}(x) \in H^{\perp}$, which is of dimension 2. But we can exercise two dimensions of freedom by adding constants to $\mu(x)$. Thus we can assume $\mu(x) \cdot i \hat{\mathbf{f}}^{\prime}(x) \equiv 0$, in other words $\mu \| \mathbf{f}^{\prime}$, and hence is of the form $\beta(x) \hat{\mathbf{f}}^{\prime}(x)$.

We will consider the operators $A_{1}, A_{2} \in \mathcal{L}\left(C_{0}\left(\mathbb{R}^{2}, \mathbb{R}^{2}\right), \mathbb{R}^{2}\right)$ defined by:

$$
\begin{aligned}
& A_{1} \mathbf{U}:=\iint_{(\mathbb{R} / 2 \pi)^{2}} t(x, y)(\mathbf{f}(x)-\mathbf{f}(y)) \int_{\mathbf{f}(y)}^{\mathbf{f}(x)} \mathbf{U} \cdot \mathbf{d} s, \\
& A_{2} \mathbf{U}:=\int_{\mathbb{R} / 2 \pi} \beta(x) \hat{\mathbf{f}}^{\prime}(x)\left[\mathbf{U}(\mathbf{f}(x)) \cdot \mathbf{f}^{\prime}(x)\right] d x .
\end{aligned}
$$

Since $A_{1}$ and $A_{2}$ are linear combinations of projections, they are symmetric; that is, there exist $a_{j}, b_{j}, e_{j} \in \mathcal{L}\left(C_{0}\left(\mathbb{R}^{2}, \mathbb{R}\right), \mathbb{R}\right)=C_{0}\left(\mathbb{R}^{2}\right)^{*}$ such that $A_{j}=\left(\begin{array}{ll}a_{j} & b_{j} \\ b_{j} & e_{j}\end{array}\right)$. Then $A:=A_{1}-A_{2}=\left(\begin{array}{ll}a & b \\ b & e\end{array}\right)$, where $a, b, e \in \mathcal{L}\left(C_{0}\left(\mathbb{R}^{2}, \mathbb{R}\right), \mathbb{R}\right)=C_{0}\left(\mathbb{R}^{2}\right)^{*}$. We have:

$$
\begin{aligned}
A_{1} \nabla g & =\iint_{(\mathbb{R} / 2 \pi)^{2}} t(x, y)(\mathbf{f}(x)-\mathbf{f}(y))(g(\mathbf{f}(x))-g(\mathbf{f}(y))) \\
& =\left(\left\langle t, R\left(\mathbf{e}_{1} g(\mathbf{f}(\cdot))\right)\right\rangle,\left\langle t, R\left(\mathbf{e}_{2} g(\mathbf{f}(\cdot))\right)\right\rangle\right) \\
& =\int_{\mathbb{R} / 2 \pi} \beta(x) \hat{\mathbf{f}}^{\prime}(x)\left[\nabla g(\mathbf{f}(x)) \cdot \mathbf{f}^{\prime}(x)\right] d x=A_{2} \nabla g .
\end{aligned}
$$

Hence $A \nabla g=0$ for all $g \in C_{0}^{\infty}\left(\mathbb{R}^{2}\right)$.

By the generalization of the Maxwell-Cremona Theorem, Theorem 5.1, there exists a $c \in C_{c}\left(\mathbb{R}^{2}\right)$ such that we have (in the distributional sense):

$$
A \mathbf{U}=\iint_{\mathbb{R}^{2}}\left(\begin{array}{rr}
c_{y y} & -c_{x y} \\
-c_{x y} & c_{x x}
\end{array}\right) \mathbf{U} d x d y
$$

Now the matrices $\left(\begin{array}{cc}c_{y y} & -c_{x y} \\ -c_{x y} & c_{x x}\end{array}\right)$ and $\left(\begin{array}{cc}c_{x x} & c_{x y} \\ c_{x y} & c_{y y}\end{array}\right)$ are related by a similarity transform. The former is a positive linear combination of projections at every point in $\mathbb{R}^{2}-$ $\mathbf{f}(\mathbb{R} / 2 \pi)$; hence the latter is positive at every point not on the curve as well. Hence $c$ is locally convex on the interior of the curve and on the exterior of the curve.

Now let $M=\sup _{\mathbf{p} \in \mathbb{R}^{2}} c(\mathbf{p})$ and define the nonempty closed set $S=\left\{\mathbf{p} \in \mathbb{R}^{2}\right.$ : $c(\mathbf{p})=M\}$. Suppose $\mathbf{p} \in \partial S$ and $\mathbf{p} \notin \mathbf{f}(\mathbb{R} / 2 \pi)$. Then there is a neighborhood of $\mathbf{p}$ which is disjoint from $\mathbf{f}(\mathbb{R} / 2 \pi)$. In this neighborhood, $c$ will be convex. Hence the whole neighborhood will belong to $S$, a contradiction. Thus $\partial S \subseteq \mathbf{f}(\mathbb{R} / 2 \pi)$. We thus have four cases:

(1) $S$ is the closure of the exterior of the curve.

(2) $S$ is the closure of the interior of the curve.

(3) $S$ is a closed subset of the curve.

(4) $S$ is the whole plane.

If (1) is true, then $c$ is zero on the curve. This implies that $\mathbf{f}$ is a level curve of a function with positive Hessian and as such must be convex. If (4) is true, then $c \equiv 0$. Then for every $(x, y) \in \operatorname{supp} t$, we will necessarily have the line segment joining $\mathbf{f}(x)$ and $\mathbf{f}(y)$ completely contained in $\mathbf{f}(\mathbb{R} / 2 \pi)$. This is because if not, then 
there would be a point in $\mathbb{R}^{2}-\mathbf{f}(\mathbb{R} / 2 \pi)$ where the matrix $\left(\begin{array}{cc}c_{y y} & -c_{x y} \\ -c_{x y} & c_{x x}\end{array}\right)$ would be positive, giving $c$ upward convexity. Case (2) is easily disposed of since $c=0$ outside the convex hull of the curve and hence will be zero on at least one point of the curve. Hence the maximum value $c$ attains is zero, a contradiction. Thus it suffices to show that case (3) cannot happen.

Assume (3) is true. We have two cases:

$\left(1^{\prime}\right)$ There exists $x \in \mathbb{R} / 2 \pi$ such that for every $\delta>0, \mathbf{f}([x, x+\delta]) \nsubseteq S$ and $\mathbf{f}([x-\delta, x]) \nsubseteq S$

$\left(2^{\prime}\right)$ There does not exist such an $x \in \mathbb{R} / 2 \pi$.

We will deal with the easier case $\left(1^{\prime}\right)$ first. WLOG $x=0$. Also, WLOG, f $(x)$. $\mathbf{e}_{1}$ is one to one for $|x|<\epsilon$. Choose $\delta_{1}, \delta_{2}>0$ such that the curve in the square $\left[-\delta_{1}, \delta_{1}\right] \times\left[-\delta_{2}, \delta_{2}\right] \subset \mathbb{R}^{2}$ looks like the graph of a function, that is, $\mathbf{f}^{-1}\left(\left[-\delta_{1}, \delta_{1}\right] \times\left[-\delta_{2}, \delta_{2}\right]\right) \subseteq[-\epsilon, \epsilon]$. Let $-\delta_{1}<x_{-}<0<x_{+}<\delta_{1}$ have $c\left(x_{-}, 0\right) \neq M$ and $c\left(x_{+}, 0\right) \neq M$. Now let:

$$
M^{\prime}=\frac{1}{2}\left(M+\max _{\mathbf{p} \in \partial\left[x_{-}, x_{+}\right] \times\left[-\delta_{2}, \delta_{2}\right]} c(\mathbf{p})\right)<M .
$$

Let $y_{+}$be the least $y>0$ such that $c(0, y)=M^{\prime}$ and let $y_{-}$be the highest $y<0$ such that $c(0, y)=M^{\prime}$. Consider the level curves passing through $y_{+}$and $y_{-}$. By the convexity of $c$ they must curve away from $(0,0)$ where the maximum occurs, but they must meet the curve on both sides of $(0,0)$ at some $x_{-}^{\prime}$ and $x_{+}^{\prime}$. This is a contradiction.

Now suppose $\left(2^{\prime}\right)$ is true. Let $[x, y] \subset \mathbb{R} / 2 \pi$ satisfy $c(\mathbf{f}([x, y]))=M$ and for every $\delta>0, \mathbf{f}([x-\delta, x]) \nsubseteq S$ and $\mathbf{f}([y, y+\delta]) \nsubseteq S$. Then $\mathbf{f}([x, y])$ is a level curve of $c$ restricted to the interior of the curve. As the level curve of a convex function it must be curved towards the interior of the curve. But by the same reasoning, $\mathbf{f}([x, y])$ is a level curve of $c$ restricted to the outside of the curve, and hence must be curved towards the outside of the curve. Hence $\mathbf{f}([x, y])$ is a line segment. As above, we can rotate $\mathbf{f}$ so it looks like the graph of a function $\mathbb{R} \longrightarrow \mathbb{R}$ near $\mathbf{f}(x)$ and near $\mathbf{f}(y)$. Using the same procedure as above, we get a contradiction by considering level curves of $M-\eta$ for a suitably small $\eta>0$.

We have now justified every step in the proof of Theorem 3.4 except for Proposition 3.7 and the generalized Maxwell-Cremona Theorem. We will prove these next.

\section{A generalization of the Farkas Lemma}

The Farkas Lemma from linear programming is as follows:

Lemma 4.1 (Farkas Lemma). Let $A: \mathbb{R}^{n} \rightarrow \mathbb{R}^{m}$ be a linear transformation. Then exactly one of the following two statements holds:

(1) There exists a nonzero $y \in \mathbb{R}^{m}$ whose components are all nonnegative and which satisfies $A^{\mathrm{T}} y=0$.

(2) There exists an $x \in \mathbb{R}^{n}$ such that every component of $A x$ is positive.

The generalization of the Farkas Lemma that we will need will have the basic form:

Theorem 4.2. Let $X$ be a compact Hausdorff space and $Y$ a (real) Hilbert space. Let $A: Y \longrightarrow C(X)$ be linear and bounded. Also let $A^{\prime}: C(X)^{*} \longrightarrow Y$ denote 
its adjoint, that is, $\langle\lambda, A y\rangle=\left\langle A^{\prime} \lambda, y\right\rangle$. Then exactly one of the following two statements holds:

(1) There exists a nonzero positive $t \in C(X)^{*}$ such that $A^{\prime} t=0$.

(2) There exists a $y \in Y$ such that $A y>0$.

We remark that if we take $Y$ to be finite dimensional and $X$ to consist of a finite number of points, then we recover Lemma 4.1 .

Proof. It is trivial that (1) and (2) cannot simultaneously hold, for if so, $0=$ $\left\langle A^{\prime} t, y\right\rangle=\langle t, A y\rangle>0$.

It remains to show that $\sim(1) \Longrightarrow(2)$. Let $T:=\left\{t \in C(X)^{*}: t \geq 0\right\}$.

We claim that there exists $\epsilon>0$ such that $\left\|A^{\prime} t\right\| \geq \epsilon\|t\|$ for all $t \in T$. If we suppose the contrary, then there exists a sequence $t_{n} \in T$ with $\left\|t_{n}\right\|=1$ such that $A^{\prime} t_{n} \rightarrow 0$. By the Banach-Alaoglu Theorem, there exists a subnet $t_{\alpha}$ which converges to $t \in T$ (in the weak-* topology on $T$ ). We know that we will have $t \in T$ and $\|t\|=1$. Also, for all $y \in Y$, we have:

$$
0=\lim _{\alpha}\left\langle A^{\prime} t_{\alpha}, y\right\rangle=\lim _{\alpha}\left\langle t_{\alpha}, A y\right\rangle=\langle t, A y\rangle=\left\langle A^{\prime} t, y\right\rangle .
$$

Thus $A^{\prime} t=0$, contradicting $\sim(1)$. Thus the claim is true. We now can show (2).

Let $t_{n} \in T$ be a sequence such that $\left\|t_{n}\right\|=1$ and

$$
\left\|A^{\prime} t_{n}\right\| \rightarrow \inf _{\substack{t \in T \\\|t\|=1}}\left\|A^{\prime} t\right\|=: w \geq \epsilon .
$$

Then a subnet $t_{\alpha}$ will converge in the weak-* topology to a limit $t_{\infty}$. Now:

$$
w \leq\left\|A^{\prime} t_{\infty}\right\| \leq \liminf _{\alpha}\left\|A^{\prime} t_{\alpha}\right\|=w .
$$

Hence $\left\|A^{\prime} t_{\infty}\right\|=w$.

Let $y:=A^{\prime} t_{\infty} /\left\|A^{\prime} t_{\infty}\right\|$. We claim that $(A y)(x) \geq \epsilon$ for all $x \in X$. It suffices to show that $\langle t, A y\rangle \geq w$ for all $t \in T$ with $\|t\|=1$. But if $\langle t, A y\rangle<w$ for some $t \in T$ with $\|t\|=1$, then $\left\langle A^{\prime} t, y\right\rangle<w$. Consider then:

$$
\begin{aligned}
\left.\frac{d}{d \eta}\right|_{\eta=0} & \left\|A^{\prime}\left((1-\eta) t_{\infty}+\eta t\right)\right\|^{2} \\
& =\left.\frac{d}{d \eta}\left[(1-\eta)^{2}\left\|A^{\prime} t_{\infty}\right\|^{2}+2 \eta(1-\eta)\left\langle A^{\prime} t, A^{\prime} t_{\infty}\right\rangle+\eta^{2}\left\|A^{\prime} t\right\|^{2}\right]\right|_{\eta=0} \\
& =-2 w^{2}+2\left\langle A^{\prime} t, w y\right\rangle<0 .
\end{aligned}
$$

This is a contradiction since $\left\|(1-\eta) t_{\infty}+\eta t\right\|=1$. Hence the proof is complete.

We can prove Proposition 3.7 using the same proof outline from Theorem 4.2 . We will, however, need the following approximation lemma.

Lemma 4.3. Suppose $\mathbf{f}_{n} \rightarrow \mathbf{f}$ in $D$ and that $q \in Q_{\mathbf{f}}$ is of the form $q^{\prime}=\lambda i \mathbf{f}^{\prime}$ where $\lambda$ is smooth. Then there exist $q_{n} \in Q_{\mathbf{f}_{n}}$ such that $q_{n} \rightarrow q$ (weakly).

Proof. We will search for $q_{n}$ of the form $q_{n}^{\prime}=\left(\lambda+\nu_{n}\right) i \mathbf{f}_{n}^{\prime}$. We will have $\left\|q_{n}\right\|$ bounded if $\left\|\nu_{n}\right\|_{\infty}$ is bounded. Hence we will have $q_{n} \rightarrow q$ weakly if $\left\|\nu_{n}\right\|_{\infty}$ is 
bounded and $\left\langle\ell, q-q_{n}\right\rangle \rightarrow 0$ for all smooth $\ell \in H$. Now $\left|\left\langle\ell, q-q_{n}\right\rangle\right|$ is equal to:

$$
\begin{aligned}
\left|\int_{\mathbb{R} / 2 \pi} \ell^{\prime} \cdot\left(q^{\prime}-q_{n}^{\prime}\right)\right| & =\left|\int_{\mathbb{R} / 2 \pi} \ell^{\prime} \cdot\left(\lambda i \mathbf{f}^{\prime}-\lambda i \mathbf{f}_{n}^{\prime}-\nu_{n} i \mathbf{f}_{n}^{\prime}\right)\right| \\
& \leq\left|\int_{\mathbb{R} / 2 \pi} \ell^{\prime} \lambda \cdot i\left(\mathbf{f}^{\prime}-\mathbf{f}_{n}^{\prime}\right)\right|+\left|\int_{\mathbb{R} / 2 \pi} \nu_{n} \ell^{\prime} \cdot i \mathbf{f}_{n}^{\prime}\right| \\
& =\left|\int_{\mathbb{R} / 2 \pi}\left[\ell^{\prime \prime} \lambda+\ell^{\prime} \lambda^{\prime}\right] \cdot i\left[\mathbf{f}-\mathbf{f}_{n}\right]\right|+\left|\int_{\mathbb{R} / 2 \pi} \nu_{n} \ell^{\prime} \cdot i \mathbf{f}_{n}^{\prime}\right| \\
& \leq 2 \pi\left\|\ell^{\prime \prime} \lambda+\ell^{\prime} \lambda^{\prime}\right\|_{\infty}\left\|\mathbf{f}-\mathbf{f}_{n}\right\|_{\infty}+\left\|\nu_{n}\right\|_{\infty}\left\|\ell^{\prime}\right\|_{\infty} \sqrt{2 \pi}\left\|\mathbf{f}_{n}\right\| .
\end{aligned}
$$

By Lemma 3.1 $\left\|\mathbf{f}-\mathbf{f}_{n}\right\|_{\infty} \rightarrow 0$. Thus in order for $q_{n} \rightarrow q$ weakly, all we need is $\left\|\nu_{n}\right\|_{\infty} \rightarrow 0$ and $\int_{\mathbb{R} / 2 \pi}\left(\lambda+\nu_{n}\right) \mathbf{f}_{n}^{\prime}=0$ (because clearly we must have $\int_{\mathbb{R} / 2 \pi} q_{n}^{\prime}=0$ ). Using integration by parts, this last equality can be written:

$$
\int_{\mathbb{R} / 2 \pi} \mathbf{f}_{n} \nu_{n}^{\prime}=\int_{\mathbb{R} / 2 \pi}\left[\mathbf{f}-\mathbf{f}_{n}\right] \lambda^{\prime} .
$$

We can pick $a_{1}, a_{2}$, and $a_{3}$ in $\mathbb{R} / 2 \pi$ such that:

$$
\left|\begin{array}{ccc}
1 & 1 & 1 \\
\mathbf{f}\left(a_{1}\right) \cdot \mathbf{e}_{1} & \mathbf{f}\left(a_{2}\right) \cdot \mathbf{e}_{1} & \mathbf{f}\left(a_{3}\right) \cdot \mathbf{e}_{1} \\
\mathbf{f}\left(a_{1}\right) \cdot \mathbf{e}_{2} & \mathbf{f}\left(a_{2}\right) \cdot \mathbf{e}_{2} & \mathbf{f}\left(a_{3}\right) \cdot \mathbf{e}_{2}
\end{array}\right| \geq 2 \epsilon>0 .
$$

There exists an $N$ such that for every $n \geq N$, the determinant with $\mathbf{f}$ replaced with $\mathbf{f}_{n}$ is greater than $\epsilon$. It suffices to choose $\nu_{n}$ for $n \geq N$. Set $C_{n}=\int_{\mathbb{R} / 2 \pi}\left[\mathbf{f}-\mathbf{f}_{n}\right] \lambda^{\prime}$. We know that $\left|C_{n}\right| \leq 2 \pi\left\|\lambda^{\prime}\right\|_{\infty}\left\|\mathbf{f}-\mathbf{f}_{n}\right\|_{\infty}$. We solve the following system of equations for $b_{n, i} \in \mathbb{R}$ :

$$
\begin{aligned}
b_{n, 1}+\quad b_{n, 2}+\quad b_{n, 3} & =0 \\
\mathbf{f}_{n}\left(a_{1}\right) b_{n, 1}+\mathbf{f}_{n}\left(a_{2}\right) b_{n, 2}+\mathbf{f}_{n}\left(a_{3}\right) b_{n, 3} & =C_{n}
\end{aligned}
$$

For $n \geq N$, we can use Cramer's Rule to give the following bound on the solution:

$$
\left|b_{n, i}\right| \leq \epsilon^{-1} 2\left[2 \pi\left\|\lambda^{\prime}\right\|_{\infty}\left\|\mathbf{f}-\mathbf{f}_{n}\right\|_{\infty}\right] 2\left[\sqrt{2 \pi}\left\|\mathbf{f}_{n}\right\|\right] .
$$

Set $\nu_{n}(0)=0$ and

$$
\nu_{n}^{\prime}(x)=b_{n, 1} \delta\left(x-a_{1}\right)+b_{n, 2} \delta\left(x-a_{2}\right)+b_{n, 3} \delta\left(x-a_{3}\right) .
$$

Then we will guarantee $\int_{\mathbb{R} / 2 \pi} \nu_{n}^{\prime}=0$, equation (4.6), and $\left\|\nu_{n}\right\|_{\infty} \rightarrow 0$. Thus we will have $q_{n} \rightarrow q$ (weakly).

Proof of Proposition 3.7. We will write $V(\mathbf{f})$ for $\left\{(x, y) \in(\mathbb{R} / 2 \pi)^{2}:(\mathbf{f}, x, y) \in V\right\}$. Also, if $Z \subset(\mathbb{R} / 2 \pi)^{2}$, we will write $T_{Z}$ for $\left\{t \in C\left((\mathbb{R} / 2 \pi)^{2}\right): t \geq 0\right.$ and $\left.\operatorname{supp} t \subseteq Z\right\}$. We assume Theorem 3.6. Let $\pi_{\mathbf{f}}: H \longrightarrow Q_{\mathbf{f}}$ be the orthogonal projection and let $J_{\mathbf{f}}=\pi_{\mathbf{f}} \circ R_{\mathbf{f}}^{\prime}$. Then Theorem 3.6 implies "If $\mathbf{f} \in D_{1}, t \in T_{V(\mathbf{f})}$, and $J_{\mathbf{f}} t=0$, then $t=0 "$.

We claim that there exists $\epsilon>0$ such that $\left\|J_{\mathbf{f}} t\right\| \geq \epsilon\|t\|$ for all $\mathbf{f} \in D_{1}$ and $t \in T_{V(\mathbf{f})}$. If we suppose the contrary, then there exist two sequences, $\mathbf{f}_{n} \in D_{1}$ and $t_{n} \in T_{V\left(\mathbf{f}_{n}\right)}$ with $\left\|t_{n}\right\|=1$ such that $\left\|J_{\mathbf{f}_{n}} t_{n}\right\| \rightarrow 0$. Since $D_{1}$ is weakly closed, it is compact by the Banach-Alaoglu Theorem; hence there exists a convergent 
subsequence of $\mathbf{f}_{n}$ which we assume WLOG is the whole sequence, so that $\mathbf{f}_{n} \rightarrow \mathbf{f}$. Since this means that $\mathbf{f}_{n} \rightarrow \mathbf{f}$ uniformly, we will have $\left\|R_{\mathbf{f}_{n}}^{\prime}-R_{\mathbf{f}}^{\prime}\right\| \rightarrow 0$. Thus:

$$
\left\|\pi_{\mathbf{f}_{n}} R_{\mathbf{f}_{n}}^{\prime} t_{n}\right\| \rightarrow 0 \Longrightarrow\left\|\pi_{\mathbf{f}_{n}} R_{\mathbf{f}^{\prime}}^{\prime} t_{n}\right\| \rightarrow 0 .
$$

Now there is also a weak-* convergent subsequence of the $t_{n}$ by the Banach-Alaoglu Theorem, which again WLOG is the whole sequence. Thus $t_{n} \rightarrow t \in T_{V(\mathbf{f})}$ since $V$ is closed; also $\|t\|=1$. Pick some $q \in Q_{\mathbf{f}}$ which can be written as $q^{\prime}=\lambda i \mathbf{f}^{\prime}$ where $\lambda$ is smooth (such $q$ are dense in $Q_{\mathbf{f}}$ ). Let $q_{n} \in Q_{\mathbf{f}_{n}}$ be the sequence guaranteed to exist by Lemma 4.3. We note that since $q_{n}$ is weakly convergent, it is bounded. Now:

$$
0=\lim _{n \rightarrow \infty}\left\langle\pi_{\mathbf{f}_{n}} R_{\mathbf{f}}^{\prime} t_{n}, q_{n}\right\rangle=\lim _{n \rightarrow \infty}\left\langle R_{\mathbf{f}}^{\prime} t_{n}, q_{n}\right\rangle=\lim _{n \rightarrow \infty}\left\langle t_{n}, R_{\mathbf{f}} q_{n}\right\rangle .
$$

Now by Lemma 3.1, $R_{\mathrm{f}} q_{n} \rightarrow R_{\mathrm{f}} q$ strongly. Thus the final limit in equation (4.13) is equal to $\left\langle t, R_{\mathbf{f}} q\right\rangle$. This means that $\left\langle R_{\mathbf{f}}^{\prime} t, q\right\rangle=0$ for a dense subset of $q \in Q_{\mathbf{f}}$. Thus $J_{\mathbf{f}} t=0$ where $\mathbf{f} \in D_{1}$ and $t \in T_{V(\mathbf{f})}-\{0\}$, contradicting Theorem 3.6. Thus the claim is proved.

We can now show the existence of an appropriate $\varphi$ for every $\mathbf{f} \in D_{1}$ exactly as in the proof of Theorem 4.2.

Fix some $\mathbf{f} \in D_{1}$. Let $t_{n} \in T_{V(\mathbf{f})}$ be a sequence such that $\left\|t_{n}\right\|=1$ and

$$
\left\|J_{\mathbf{f}} t_{n}\right\| \rightarrow \inf _{\substack{t \in T_{V(\mathbf{f})} \\\|t\|=1}}\left\|J_{\mathbf{f}} t\right\|=: w \geq \epsilon
$$

A subsequence is weak-* convergent (WLOG the whole sequence) to a limit $t_{\infty}$. Using the same reasoning as above, we conclude that $J_{\mathbf{f}} t_{n} \rightarrow J_{\mathbf{f}} t_{\infty}$ in the weak topology, so:

$$
w \leq\left\|J_{\mathbf{f}} t_{\infty}\right\| \leq \liminf \left\|J_{\mathbf{f}} t_{n}\right\|=w .
$$

Thus $\left\|J_{\mathbf{f}} t_{\infty}\right\|=w$. Let $q:=J_{\mathbf{f}} t_{\infty} /\left\|J_{\mathbf{f}} t_{\infty}\right\|$.

Now we claim that $\left\langle J_{\mathbf{f}} t, q\right\rangle \geq w\|t\|$ for all $t \in T_{V(\mathbf{f})}$. Suppose not, that we have $t \in T_{V(\mathbf{f})}$ with $\|t\|=1$ and $\left\langle J_{\mathbf{f}} t, q\right\rangle<w$. Then $\left\langle J_{\mathbf{f}} t, J_{\mathbf{f}} t_{\infty}\right\rangle<w^{2}$. But consider then:

$$
\begin{aligned}
\left.\frac{d}{d \eta}\right|_{\eta=0} & \left\|J_{\mathbf{f}}\left((1-\eta) t_{\infty}+\eta t\right)\right\|^{2} \\
& =\left.\frac{d}{d \eta}\left[(1-\eta)^{2}\left\|J_{\mathbf{f}} t_{\infty}\right\|^{2}+2 \eta(1-\eta)\left\langle J_{\mathbf{f}} t, J_{\mathbf{f}} t_{\infty}\right\rangle+\eta^{2}\left\|J_{\mathbf{f}} t\right\|^{2}\right]\right|_{\eta=0} \\
& =-2 w^{2}+2\left\langle J_{\mathbf{f}} t_{\infty}, J_{\mathbf{f}} t\right\rangle<0 .
\end{aligned}
$$

This is a contradiction since $\left\|(1-\eta) t_{\infty}+\eta t\right\|=1$. Hence the claim is proved.

Let $\varphi=q$. Then:

$$
\left\langle t, R_{\mathbf{f}} \varphi\right\rangle=\left\langle J_{\mathbf{f}} t, q\right\rangle \geq w\|t\| \geq \epsilon\|t\| \text { for all } t \in T_{V(\mathbf{f})} .
$$

This means that $R_{\mathbf{f}} \varphi(x, y) \geq \epsilon$ for all $(x, y) \in V(\mathbf{f})$.

\section{A generalization of the Maxwell-Cremona Theorem}

Let $A \in \mathcal{L}\left(C_{0}\left(\mathbb{R}^{2}, \mathbb{R}^{2}\right), \mathbb{R}^{2}\right)$ have compact support. Then by the Riesz Representation Theorem, $A$ can be thought of as a matrix of measures on $\mathbb{R}^{2}$ :

$$
A=\left(\begin{array}{ll}
a & b \\
d & e
\end{array}\right) .
$$


We are concerned with the case when $A$ is symmetric, that is, $b=d$. For the moment, suppose $a, b$, and $e$ are continuous functions. In this case, at each point $A$ has orthogonal eigenvectors $\mathbf{v}_{1}$ and $\mathbf{v}_{2}$ with eigenvalues $\lambda_{1}$ and $\lambda_{2}$. We think of $A$ as representing a "stress" on the plane, where at each point, there is tension in the $\mathbf{v}_{i}$ direction of magnitude $\lambda_{i}$. It turns out that it is right to call such a stress an "equilibrium stress" if:

$$
A \nabla g=0 \text { for all } g \in C_{0}^{\infty}\left(\mathbb{R}^{2}\right) .
$$

In the case that $a, b$, and $e$ are continuous, it is straightforward to show that in fact:

$$
A=\left(\begin{array}{ll}
a & b \\
b & e
\end{array}\right)=\left(\begin{array}{rr}
c_{y y} & -c_{x y} \\
-c_{x y} & c_{x x}
\end{array}\right)
$$

The function $c$ will be in $C_{c}\left(\mathbb{R}^{2}\right)$. This is the Maxwell-Cremona "lifting" of the stress represented by $A$.

However, the notion of being an equilibrium stress (5.2) makes sense for any compactly supported $A$, so one would expect that (5.3) should hold in some sense for all equilibrium stresses $A$. If $\mathbf{U}$ is a smooth vector field and we integrate $\iint_{\mathbb{R}^{2}}\left(\begin{array}{cc}c_{y y} & -c_{x y} \\ -c_{x y} & c_{x x}\end{array}\right) \mathbf{U} d x d y$ by parts, we get $\iint_{\mathbb{R}^{2}} c[i \nabla \operatorname{curl} \mathbf{U}] d x d y$, so if (5.3) holds in the distributional sense, we would like this last integral to give $A \mathbf{U}$ for smooth $\mathbf{U}$. This is the intuition for the following theorem.

Theorem 5.1. Let $A \in \mathcal{L}\left(C_{0}\left(\mathbb{R}^{2}, \mathbb{R}^{2}\right), \mathbb{R}^{2}\right)$ have compact support. Suppose $A$ is symmetric; that is, there exist $a, b, c \in C_{0}\left(\mathbb{R}^{2}\right)^{*}$ such that:

$$
A=\left(\begin{array}{ll}
a & b \\
b & e
\end{array}\right) .
$$

Additionally, suppose that for every $g \in C_{0}^{\infty}\left(\mathbb{R}^{2}\right), A \nabla g=0$. Then there exists $c \in C_{c}\left(\mathbb{R}^{2}\right)$ such that for all $\mathbf{U} \in C_{0}^{\infty}\left(\mathbb{R}^{2}, \mathbb{R}^{2}\right)$ :

$$
A \mathbf{U}=\iint_{\mathbb{R}^{2}} c[i \nabla \operatorname{curl} \mathbf{U}] d x d y
$$

Proof. First, let us show that (the matrix of measures associated with) $A$ has no pure point part. Let $\mathbf{p}$ and $\mathbf{v}$ be arbitrary. Choose $g \in C_{0}^{\infty}\left(\mathbb{R}^{2}\right)$ so that $\nabla g(\mathbf{p})=\mathbf{v}$. Then $0=A(\nabla g)(\epsilon(\cdot)+\mathbf{p})$, but as $\epsilon \rightarrow 0$, the right hand side approaches the pure point part of $A$ at $\mathbf{p}$ applied to $\mathbf{v}$. Hence $A$ has no pure point part.

Consider the measure $|A| \in C_{0}\left(\mathbb{R}^{2}\right)^{*}$, where the $|\cdot|$ of a matrix is its operator norm. In other words, for $f \geq 0$, we define:

$$
|A| f:=\sup _{\substack{\theta: \mathbb{R}^{2} \longrightarrow \mathbb{R} \\
\psi: \mathbb{R}^{2} \longrightarrow \mathbb{R}}} \iint_{\mathbb{R}^{2}}\left(\begin{array}{ll}
\cos \theta & \sin \theta
\end{array}\right)\left(\begin{array}{ll}
a & b \\
b & e
\end{array}\right)\left(\begin{array}{c}
\cos \psi \\
\sin \psi
\end{array}\right) f .
$$

We know $|A|$ comes from a measure, which we will also denote $|A|$. Let $\mu(\theta)$ be the measure on the real line $\mathbb{R}$ at angle $\theta$ passing through the origin, obtained by 
projecting the measure $|A|$ orthogonally onto the line. In other words:

$$
\int_{\mathbb{R}} f(x) d \mu(\theta)=\iint_{\mathbb{R}^{2}} f((x, y) \cdot(\cos \theta, \sin \theta))|A| .
$$

Now let $\mu_{\mathrm{pp}}(\theta)$ be the pure point part of $\mu(\theta)$. I claim that $\mu_{\mathrm{pp}}(\theta) \neq 0$ for at most countably many $\theta$. We note that this is implied by the following:

$$
\sum_{i=1}^{N}\left\|\mu_{\mathrm{pp}}\left(\theta_{i}\right)\right\| \leq\||A|\| \text { whenever the } \theta_{i} \text { are distinct. }
$$

But (5.8) is true because any part of $|A|$ which contributes to both $\left\|\mu_{\mathrm{pp}}\left(\theta_{i}\right)\right\|$ and $\left\|\mu_{\mathrm{pp}}\left(\theta_{j}\right)\right\|$ would have to be supported on a countable set of points, and hence would have to be pure point, which we know $A$, and hence $|A|$ does not have. Now let $m(\theta, h)=\sup _{x \in \mathbb{R}} \int_{x}^{x+h} \mu(\theta)(y) d y$. Now $m(\theta, h) \rightarrow 0$ as $h \rightarrow 0$ if $\mu(\theta)$ has no pure point part; thus $m(\theta, h) \rightarrow 0$ for almost all $\theta$. This fact being proved, we can proceed to the construction of $c$.

Let $\phi$ be a smooth real-valued even function on $\mathbb{R}^{2}$ with support contained in the unit disc which satisfies $\phi \geq 0$ and $\iint_{\mathbb{R}^{2}} \phi=1$. Let $\phi_{\eta}(\mathbf{p})=\eta^{-2} \phi\left(\eta^{-1} \mathbf{p}\right)$. We can then define the operator:

$$
A_{\eta}=A * \phi_{\eta}=\left(\begin{array}{ll}
a^{(\eta)} & b^{(\eta)} \\
b^{(\eta)} & e^{(\eta)}
\end{array}\right) .
$$

Now we know that:

$$
a^{(\eta)}, b^{(\eta)}, e^{(\eta)} \in C_{0}^{\infty}\left(\mathbb{R}^{2}\right) \text { and that } A_{\eta} \nabla g=0 \text { for all } g \in C_{0}^{\infty}\left(\mathbb{R}^{2}\right) .
$$

Thus the vector fields $\left(a^{(\eta)}, b^{(\eta)}\right)$ and $\left(b^{(\eta)}, e^{(\eta)}\right)$ have zero divergence. That means there exist $f^{(\eta)}, g^{(\eta)} \in C_{0}^{\infty}\left(\mathbb{R}^{2}\right)$ such that $a^{(\eta)}=f_{y}^{(\eta)}, b^{(\eta)}=-f_{x}^{(\eta)}=-g_{y}^{(\eta)}$, and $e^{(\eta)}=g_{x}^{(\eta)}$. The equality $f_{x}^{(\eta)}=g_{y}^{(\eta)}$ implies that there exists $c^{(\eta)} \in C_{0}^{\infty}\left(\mathbb{R}^{2}\right)$ such that $f^{(\eta)}=c_{y}^{(\eta)}$ and $g^{(\eta)}=c_{x}^{(\eta)}$. In other words:

$$
A_{\eta}=\left(\begin{array}{rr}
c_{y y}^{(\eta)} & -c_{x y}^{(\eta)} \\
-c_{x y}^{(\eta)} & c_{x x}^{(\eta)}
\end{array}\right) .
$$

Claim: For every $\epsilon>0$, there exist $\delta>0$ and $\eta_{0}>0$ such that:

$$
\eta_{0}>\eta>0 \text { and }|\mathbf{q}-\mathbf{p}|<\delta \Longrightarrow\left|c^{(\eta)}(\mathbf{p})-c^{(\eta)}(\mathbf{q})\right|<\epsilon .
$$

Let $\epsilon>0$ be given. Suppose $\mathbf{p}=\left(x_{0}, y\right) \in \mathbb{R}^{2}$ and $\mathbf{q} \in \mathbb{R}^{2}$ and we wish to bound $\left|c^{(\eta)}(\mathbf{p})-c^{(\eta)}(\mathbf{q})\right|$ given $|\mathbf{q}-\mathbf{p}|<\delta$. To simplify notation, we will for the moment assume that $\mathbf{q}=(x, y)$. Then:

$$
\begin{aligned}
\left|c^{(\eta)}(\mathbf{p})-c^{(\eta)}(\mathbf{q})\right| & =\left|\int_{x_{0}}^{x} c_{x}^{(\eta)}(t, y) d t\right|=\left|\int_{x_{0}}^{x} \int_{-\infty}^{y} c_{x y}^{(\eta)}(t, z) d z d t\right| \\
& \leq \int_{x_{0}}^{x} \int_{-\infty}^{\infty}\left|b^{(\eta)}(t, z)\right| d z d t \leq \int_{x_{0}-\eta}^{x+\eta} \int_{-\infty}^{\infty}|A(t, z)| d z d t \\
& \leq m(0, \delta+2 \eta) .
\end{aligned}
$$

Similarly, if $\theta_{\mathbf{p}}^{\mathbf{q}}$ is the angle of the segment from $\mathbf{p}$ to $\mathbf{q}$, then we have:

$$
\left|c^{(\eta)}(\mathbf{p})-c^{(\eta)}(\mathbf{q})\right| \leq m\left(\theta_{\mathbf{p}}^{\mathbf{q}}, 2 \eta+\delta\right) .
$$


Now since $m(\theta, h) \rightarrow 0$ as $h \rightarrow 0$ for all but at most countably many $\theta$, there exists $h>0$ such that the measure of the set $\{\theta: m(\theta, h)<\epsilon / 4\}$ is more than $\frac{5 \pi}{3}$. Then if $2 \eta+\delta<\min (\epsilon /(4 \pi\|t\|), h)$ and the slope of the segment from $\mathbf{p}$ to $\mathbf{q}$ is not in the exceptional set of $\theta$ (which has measure less than $\frac{\pi}{3}$ ), then $\left|c^{(\eta)}(\mathbf{p})-c^{(\eta)}(\mathbf{q})\right| \leq \epsilon / 2$. But for any $\mathbf{p}$ and $\mathbf{q}$ within $\delta$ of each other, we can find an $\mathbf{r}$ within $\delta$ of both $\mathbf{p}$ and $\mathbf{q}$ so that neither of the segments $\mathbf{p}$ to $\mathbf{r}$ and $\mathbf{r}$ to $\mathbf{q}$ are in the exceptional set of $\theta$. Hence by the triangle inequality, $\left|c^{(\eta)}(\mathbf{p})-c^{(\eta)}(\mathbf{q})\right| \leq \epsilon$ if we set $\eta_{0}=\delta=\frac{1}{4} \min (\epsilon /(4 \pi\|t\|), h)$. Thus the claim is true.

Now by the Arzelà-Ascoli Theorem, there exists a subsequence of $c^{(1 / n)}$ which converges uniformly to a continuous function $c \in C_{c}\left(\mathbb{R}^{2}\right)$. Thus let $\eta_{i} \rightarrow 0$ and satisfy $c^{\left(\eta_{i}\right)} \rightarrow c \in C_{c}\left(\mathbb{R}^{2}\right)$ uniformly as $i \rightarrow \infty$. As remarked before, if $\mathbf{U}$ is a smooth compactly supported vector field, then it is a straightforward integration by parts to show:

$$
A\left(\mathbf{U} * \phi_{\eta_{i}}\right)=A_{\eta_{i}} \mathbf{U}=\iint_{\mathbb{R}^{2}} c^{\left(\eta_{i}\right)}[i \nabla \operatorname{curl} \mathbf{U}] d x d y .
$$

Taking the limit as $i \rightarrow \infty$, we obtain (5.5) as was to be shown.

\section{OPEN PROBLEMS}

Now, we can state some conjectures on the possible strengthening of Theorem 2.2. For example, we can conjecture that there exists an $\mathbf{h}$ which is not only continuous, but in fact smooth. Also, if the initial curve is smooth, we can require that the curve be smooth at every time during the deformation.

Conjecture 6.1. Given a unit speed simple closed curve $\mathbf{f}: \mathbb{R} / 2 \pi \longrightarrow \mathbb{C}$, there exists a smooth function $\mathbf{h}:[0,1] \longrightarrow \mathcal{D}$ satisfying (1)-(4).

Conjecture 6.2. Given a smooth unit speed simple closed curve $\mathbf{f}: \mathbb{R} / 2 \pi \longrightarrow \mathbb{C}$, there exists a continuous function $\mathbf{h}:[0,1] \longrightarrow \mathcal{D}$ satisfying (1)-(4) as well as:

(5) $\mathbf{h}(t)(x)$ is a smooth function of $x$ for all $t \in[0,1]$.

We also conjecture that it is possible to extend Corollary 3.2 to something resembling the following.

Conjecture 6.3. Suppose $\mathbf{f}: \mathbb{R} / 2 \pi \longrightarrow \mathbb{R}^{2}$ is a rectifiable simple closed curve which is not convex. Then there exists $\varphi: \mathbb{R} / 2 \pi \longrightarrow \mathbb{R}^{2}$ which is absolutely continuous and satisfies $\mathbf{f}^{\prime} \cdot \varphi^{\prime} \equiv 0$, as well as $(\mathbf{f}(x)-\mathbf{f}(y)) \cdot(\varphi(x)-\varphi(y))>0$ whenever the line segment connecting $\mathbf{f}(x)$ and $\mathbf{f}(y)$ is not completely contained in $\mathbf{f}(\mathbb{R} / 2 \pi)$.

Of course, this would be in preparation to prove:

Meta-Conjecture 6.4. There exists a proof of Theorem 2.2 which does not rely on approximation by polygons.

\section{REFERENCES}

1. Jason H. Cantarella, Erik D. Demaine, Hayley N. Iben, and James F. O'Brien, An energy-driven approach to linkage unfolding, SCG '04: Proceedings of the twentieth annual symposium on Computational Geometry (New York, NY, USA), ACM Press, 2004, pp. 134-143.

2. Robert Connelly, Erik D. Demaine, and Günter Rote, Straightening polygonal arcs and convexifying polygonal cycles, Discrete Comput. Geom. 30 (2003), no. 2, 205-239, U.S.Hungarian Workshops on Discrete Geometry and Convexity (Budapest, 1999/Auburn, AL, 2000). MR2007962 (2004h:52028) 
3. Mohammad Ghomi, Classical open problems in differential geometry, 2004.

4. O. Hernandez-Lerma and J. B. Lasserre, Cone-constrained linear equations in Banach spaces, J. Convex Anal. 4 (1997), no. 1, 149-164. MR1459886 (98i:90125)

Durham Academy Upper School, 3601 Ridge Road, Durham, North Carolina 27705

Current address: Department of Mathematics, Princeton University, Princeton, New Jersey 08544

E-mail address: jpardon@princeton.edu 